\title{
Neurotoxic/neuroprotective profile of carbamazepine, oxcarbazepine and two new putative antiepileptic drugs, BIA 2-093 and BIA 2-024
}

\author{
António F. Ambrósio a , Ana P. Silva a , Inês Araújo a , João O. Malva a,b, \\ Patrício Soares-da-Silva ${ }^{c}$, Arsélio P. Carvalho ${ }^{a}$, Caetana M. Carvalho ${ }^{\mathrm{a}, *}$ \\ a Department of Cell Biology, Center for Neuroscience of Coimbra, University of Coimbra, 3004-517 Coimbra, Portugal \\ ${ }^{\mathrm{b}}$ Laboratory of Biochemistry, Faculty of Medicine, University of Coimbra, 3004-517 Coimbra, Portugal \\ ${ }^{\mathrm{c}}$ Department of Research and Development, BIAL, 4785 S. Mamede do Coronado, Portugal
}

Received 13 April 2000; received in revised form 22 August 2000; accepted 24 August 2000

\begin{abstract}
We investigated and compared the toxicity profile, as well as possible neuroprotective effects, of some antiepileptic drugs in cultured rat hippocampal neurons. We used two novel carbamazepine derivatives, $(S)-(-)$-10-acetoxy-10,11-dihydro-5 $H$-dibenz $[b, f]$ azepine-5carboxamide (BIA 2-093) and 10,11-dihydro-10-hydroxyimino-5H-dibenz[ $b, f]$ azepine-5-carboxamide (BIA 2-024), and compared their effects with the established compounds carbamazepine and oxcarbazepine. The assessment of neuronal injury was made by using the 3-(4,5-dimethylthiazol-2-yl)-2,5-diphenyl (MTT) assay, as well as by analysing morphology and nuclear chromatin condensation (propidium iodide staining), after hippocampal neurons were exposed to the drugs for $24 \mathrm{~h}$. The putative antiepileptic drugs, BIA 2-093 or BIA 2-024 (at $300 \mu \mathrm{M}$ ), only slightly decreased MTT reduction, whereas carbamazepine or oxcarbazepine were much more toxic at lower concentrations. Treatment with the antiepileptic drugs caused nuclear chromatin condensation in some neurons, which is characteristic of apoptosis, and increased the activity of caspase-3-like enzymes, mainly in neurons treated with carbamazepine and oxcarbazepine. The toxic effect caused by carbamazepine was not mediated by $N$-methyl-D-aspartate (NMDA) or by $\alpha$-amino-3-hydroxy5-methyl-isoxazole-4-propionate (AMPA) receptors. Moreover, the antiepileptic drugs failed to protect hippocampal neurons from the toxicity caused by kainate, veratridine, or ischaemia-like conditions. (C) 2000 Elsevier Science B.V. All rights reserved.
\end{abstract}

Keywords: Antiepileptic drug; Neurotoxicity; Neuroprotection; Apoptosis

\section{Introduction}

Epilepsy is one of the most common neurological diseases, affecting at least 50 million people worldwide (Scheuer and Pedley, 1990). The major antiepileptic drugs in clinical use, i.e. phenytoin, carbamazepine, valproate and phenobarbital, among others, were developed and introduced between 1910 and 1970, and are referred to as "first-generation" drugs. Several new anticonvulsant drugs, such as vigabatrin, gabapentin, felbamate, lamotrigine and oxcarbazepine, have been introduced into clinical practice and are referred to as "second-generation" drugs. More

\footnotetext{
${ }^{*}$ Corresponding author. Tel.: +351-239-833-369; fax: +351-239-822776.

E-mail address: cmcarv@cnc.cj.uc.pt (C.M. Carvalho).
}

recent anticonvulsants, which are in preclinical or clinical development, can be considered as "third-generation" drugs (Löscher, 1998a).

Carbamazepine has become the most frequently prescribed first-line drug for the treatment of partial and generalised tonic-clonic epileptic seizures (Loiseau and Duché, 1995). Moreover, carbamazepine has been used in the treatment of neuropathic pain and schizoaffective psychosis. However, patients treated with carbamazepine may develop toxic symptoms, such as drowsiness, dizziness, ataxia and nausea. Other disadvantages of carbamazepine are its enzyme-inducing properties (Tateishi et al., 1999) and interaction with other drugs (Yasui et al., 1997), as well as the increase in the incidence of congenital malformations (Kaneko et al., 1999). Although most of the toxic symptoms of carbamazepine are in the central nervous system (CNS), the mechanisms by which this antiepileptic 
drug causes toxicity are not completely clarified. Some reports indicate that carbamazepine induces apoptosis in cultured cerebellar granule cells (Gao and Chuang, 1992; Nonaka et al., 1998) and decreases glutamine synthetase activity (Fraser et al., 1999). Conversely, it was previously shown that carbamazepine exerted protective effects against focal ischaemia or anoxia (Fern et al., 1993; Rataud et al., 1994; Minato et al., 1997), or against toxicity induced by glutamate or veratridine (Mattson and Kater, 1989; Lakics et al., 1995; Mark et al., 1995). In addition, carbamazepine did not prevent status epilepticus-induced damage to neurons in hippocampus and amygdala (Pitkänen et al., 1996).

Oxcarbazepine is an analogue of carbamazepine, with a comparable anticonvulsant efficacy. It has the advantage of a low incidence of allergic reactions and enzyme induction. In case of combination therapy with other antiepileptic drugs, oxcarbazepine is usually better tolerated than carbamazepine (Elger and Bauer, 1998). However, the involvement of oxcarbazepine in neuronal toxicity has not been investigated.

Since seizures are resistant to treatment with currently available antiepileptic drugs in about $30 \%$ of patients with epilepsy, more effective antiepileptic drugs are necessary. In addition, because of the inadequacy of the currently available antiepileptic drugs, in terms of safety, newly developed drugs should be less toxic than existing drugs. $(S)$ - $(-)$-10-acetoxy-10,11-dihydro-5 $H$-dibenz $[b, f]$ azepine-5-carboxamide (BIA 2-093; Benes et al., 1999a) and 10,11-dihydro-10-hydroxyimino-5 $H$-dibenz $[b, f]$ azepine5-carboxamide (BIA 2-024; Benes et al., 1999b) are representative of new compounds, structurally related to carbamazepine and oxcarbazepine, with anticonvulsant activity, as determined by maximal electroshock (Benes et al., 1999a,b). These two compounds were specifically designed to circumvent their further degradation to toxic metabolites, such as epoxides, without losing anticonvulsant potency. For example, BIA 2-093 produces significantly less motor and cognitive impairment than carbamazepine and oxcarbazepine (Benes et al., 1999a). In the present work, we used an in vitro system (cultured hippocampal neurons), to investigate the toxic profile of BIA 2-093 and BIA 2-024, as well as their possible protective effects against several neurotoxic insults, in comparison with the antiepileptic drugs carbamazepine and oxcarbazepine.

\section{Materials and methods}

\subsection{Cell culture}

Hippocampal neurons were dissociated from hippocampi of E18-E19 Wistar rat embryos, after treatment with trypsin $\left(1.0 \mathrm{mg} / \mathrm{ml} ; 15 \mathrm{~min} ; 37^{\circ} \mathrm{C}\right)$ and deoxyribonuclease $\mathrm{I}(0.15 \mathrm{mg} / \mathrm{ml})$ in $\mathrm{Ca}^{2+}$ - and $\mathrm{Mg}^{2+}$-free Hank's balanced salt solution ( $137 \mathrm{mM} \mathrm{NaCl}, 5.36 \mathrm{mM} \mathrm{KCl}, 0.44$ $\mathrm{mM} \mathrm{KH} \mathrm{KO}_{4}, 0.34 \mathrm{mM} \mathrm{Na} \mathrm{HPO}_{4} .2 \mathrm{H}_{2} \mathrm{O}, 4.16 \mathrm{mM}$ $\mathrm{NaHCO}_{3}, 5 \mathrm{mM}$ glucose, supplemented with $0.001 \%$ phenol red, $1 \mathrm{mM}$ sodium pyruvate, $10 \mathrm{mM}$ HEPES, $\mathrm{pH}$ 7.4). Hippocampal neurons were cultured in B27-supplemented Neurobasal medium (GIBCO), a serum-free medium combination (Brewer et al., 1993), supplemented with glutamate $(25 \mu \mathrm{M})$, glutamine $(0.5 \mathrm{mM})$ and gentamicin $(0.12$ $\mathrm{mg} / \mathrm{ml}$ ). Cultures were kept at $37^{\circ} \mathrm{C}$ in a humidified incubator in $5 \% \mathrm{CO}_{2} / 95 \%$ air, for $7-8$ days, the time required for maturation of hippocampal neurons.

For the assessment of neuronal injury with the 3-(4,5dimethylthiazol-2-yl)-2,5-diphenyltetrazolium bromide (MTT) assay, or to measure the activity of caspase-3-like enzymes, hippocampal neurons were plated on poly-Dlysine-coated $(0.1 \mathrm{mg} / \mathrm{ml})$ multiwells at a density of $0.1 \times$ $10^{6}$ or $0.2 \times 10^{6}$ cells $/ \mathrm{cm}^{2}$, respectively. For morphology studies with Cresyl violet staining, or analysis of nuclear condensation/fragmentation with propidium iodide staining, cells were plated at a density of $45 \times 10^{3}$ cells $/ \mathrm{cm}^{2}$ on poly-D-lysine-coated coverslips.

\subsection{Exposure of hippocampal neurons to drugs}

The hippocampal neurons were exposed to drugs for 24 h. The drugs were diluted in aliquots of $250 \mu l$ of conditioned medium, taken from each well and placed in an Eppendorf tube. Then, the aliquots were added back to the corresponding well, and the medium was mixed gently to preserve the integrity of neurons. The toxicity was assessed by using either the MTT assay, by analysing neuronal morphology, or by counting neurons with nuclear condensation and/or fragmentation. The activity of caspase-3-like enzymes was also measured, as described in the next.

\subsection{MTT assay}

Assessment of neuronal injury in cultured hippocampal neurons was made by using the MTT assay. Briefly, Krebs buffer with MTT $(0.5 \mathrm{mg} / \mathrm{ml})$ was added to the cultures and incubated for $1 \mathrm{~h}$ at $37^{\circ} \mathrm{C}$ in the incubation chamber. MTT, when taken up by living cells, is converted from a yellow to a water-insoluble blue-coloured product. The precipitated dye was dissolved in $0.04 \mathrm{M} \mathrm{HCl}$ in isopropanol and colorimetrically (absorbance at $570 \mathrm{~nm}$ ) quantitated.

\subsection{Morphology studies}

After incubation of cultured hippocampal neurons with the drugs, for $24 \mathrm{~h}$, the culture medium was removed, the cells were washed two times with phosphate-buffered saline (PBS; $137 \mathrm{mM} \mathrm{NaCl}, 2.7 \mathrm{mM} \mathrm{KCl}, 10 \mathrm{mM} \mathrm{Na}_{2} \mathrm{HPO}_{4}, 1.8$ $\mathrm{mM} \mathrm{KH} \mathrm{PO}_{4}, \mathrm{pH}$ 7.4), and fixed with $0.1 \%$ glutaralde- 
hyde in $\mathrm{PBS}$ at $37^{\circ} \mathrm{C}$. After $30 \mathrm{~min}$, the cells were washed two times with PBS (10 min each time) and then dehydrated in a gradient of ethanol/PBS $(50 \%, 70 \%, 80 \%$, $95 \%$ and $100 \%$ ethanol) and ethanol/acetone $1: 1$, about 1 min per solution. After this procedure, the cells were rehydrated in a gradient of ethanol/PBS (100\%, 95\%, $80 \%, 70 \%$ and $50 \%$ ethanol) and then washed two times with PBS. The cells were stained with $0.5 \%$ Cresyl violet for $5 \mathrm{~min}$, washed several times with ultrapurified water, and dried. Finally, the coverslips containing the cells were mounted with Entellan (Merck), and micrographs were taken.

\subsection{Propidium iodide staining}

After incubation with the drugs, cells were fixed in $4 \%$ paraformaldehyde and then stained with propidium iodide (1.3 $\mu \mathrm{g} / \mathrm{ml})$ in Krebs medium. Apoptotic neurons, with condensed/fragmented nuclei, were counted by using a fluorescence microscope and a triple filter (Omega Optical XF63). The neuronal counting was done by counting approximately 200 neurons in about 10 fields in each coverslip.

\subsection{Caspase-3-like activity assay}

After the exposure of hippocampal neurons to drugs for $24 \mathrm{~h}$, the culture medium was aspirated, and the cultures were washed two times with the following buffer: $50 \mathrm{mM}$ $\mathrm{KCl}, 50 \mathrm{mM}$ PIPES, $10 \mathrm{mM}$ EGTA and $2 \mathrm{mM} \mathrm{MgCl}{ }_{2}, \mathrm{pH}$ 7.4 , at $4^{\circ} \mathrm{C}$. The cells were again washed with the washing buffer supplemented with $100 \mu \mathrm{M}$ phenyl-methyl-sulfonyl-fluoride, $1 \mathrm{mM}$ dithiothreitol and $1 \mu \mathrm{g} / \mathrm{ml}$ Chymostatin-Leupeptin-Antiparin-Pepstatin A (CLAP). After this procedure, cells were lysed at $4^{\circ} \mathrm{C}$ with the previous supplemented buffer, with $0.5 \%$ Triton, collected and frozen/thawed at $-80^{\circ} \mathrm{C}$ (three times). Protein concentration was determined, and $20 \mu \mathrm{g}$ of protein was used for each determination. The extract $(20 \mu \mathrm{g})$ was added to 40 $\mu 1$ of reaction buffer (25 mM HEPES, 0.1\% 3-[(3cholamidopropyl)dimethylammonio]-1-propane-sulfonate (CHAPS), $10 \mathrm{mM}$ dithiothreitol, $100 \mu \mathrm{M}$ phenyl-methylsulfonyl-fluoride, $\mathrm{pH}$ 7.5, which was supplemented with $100 \mu \mathrm{M}$ Asp-Glu-Val-Asp-7-amino-4-trifluoromethyl coumarin (DEVD-AFC), a fluorogenic substrate for caspase- 3 and related proteases) and incubated for $30 \mathrm{~min}$, at $37^{\circ} \mathrm{C}$.
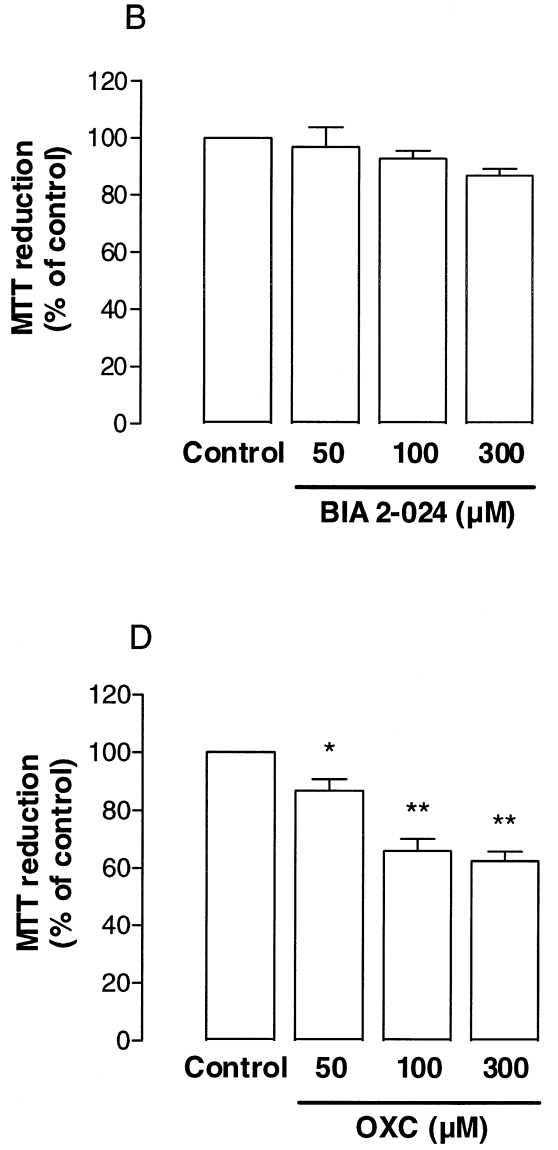

Fig. 1. Neurotoxic effect caused by exposure of cultured hippocampal neurons to antiepileptic drugs for 24 h. (A) BIA 2-093 (B) BIA 2-024 (C) Carbamazepine (CBZ) (D) Oxcarbazepine (OXC). The concentrations of the antiepileptic drugs are indicated below the bars. The results are presented as percentage of MTT reduction, compared to control conditions (no drug), and represent the means \pm S.E.M. of at least three independent experiments performed in triplicate. ${ }^{*} P<0.05,{ }^{*} P<0.01-$ Significantly different from control; Dunnett's post-test. 
After incubation, the fluorescence was monitored (excitation from 300 to $410 \mathrm{~nm}$ and emission $475 \mathrm{~nm}$ ) using a Spex Fluoromax spectrofluorometer. The results are expressed as percentage of control (no treatment), using arbitrary fluorescence units, at an excitation wavelength of $390 \mathrm{~nm}$.

\subsection{Ischemia-like conditions experiments}

Hippocampal neurons were exposed to Krebs medium without glucose and supplemented with 2-deoxyglucose (5 $\mathrm{mM})$ and sodium cyanide $(2.5 \mathrm{mM})$, which inhibits cytochrome $c$ oxidase, for $10 \mathrm{~min}$. After this period, neurons were washed two times with Krebs medium with glucose and incubated with Neurobasal medium in an incubation chamber at $37^{\circ} \mathrm{C}$, for a 1 - or 2 -h recovery period. The antiepileptic drugs tested were present during the ischaemic period and during the recovery period. After this period, the metabolic activity was assessed by using the MTT assay.

\subsection{Chemicals}

BIA 2-093, BIA 2-024, carbamazepine and oxcarbazepine were obtained from BIAL, S. Mamede do Coronado, Portugal. (-)-1-(4-Aminophenyl)-4-methyl-7,8-methylenedioxy-4,5-dihydro-3-methylcarbamoyl-2,3-benzodiazepine (LY 303070) was a kind gift of Lilly Research Laboratories, Indianapolis, IN, USA, and dizocilpine (MK801) was a kind gift of Merck Sharp and Dohme, NJ, USA. Neurobasal medium, B27 supplement, gentamicin and trypsin (USP grade) were purchased from GIBCO BRL, Life Technologies, Scotland. Glutamate, DNase (DN-25), veratridine, 2-deoxyglucose, sodium cyanide, phenyl-methyl-sulfonyl-fluoride, CLAP and dithiothreitol were purchased from Sigma, St. Louis, MO, USA. Kainate was purchased from TOCRIS, Bristol, UK. Glutaraldehyde and paraformaldehyde were obtained from MerckSchuchardt, Germany. Propidium iodide was purchased from Molecular Probes, Leiden, The Netherlands. All other reagents were from Sigma or from Merck-Schuchardt.
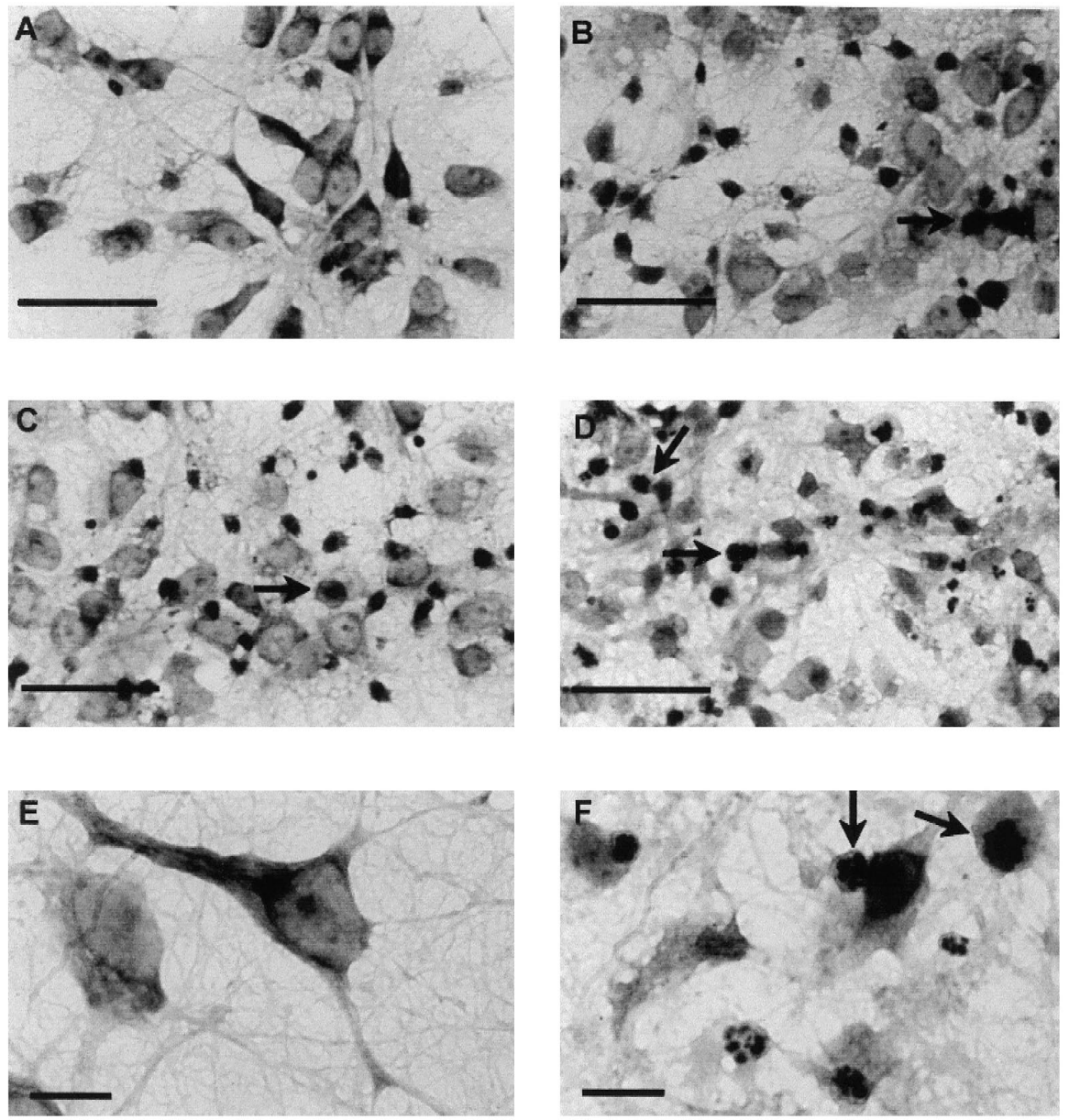

Fig. 2. Changes in the morphology of cultured hippocampal neurons treated with antiepileptic drugs for 24 h. (A) Control culture (B) $300 \mu M$ BIA 2-093 (C) $300 \mu \mathrm{M}$ carbamazepine (D) $300 \mu \mathrm{M}$ oxcarbazepine $-400 \times$ magnification; (E) Control (F) $300 \mu \mathrm{M}$ oxcarbazepine $-1000 \times$ magnification. The morphological aspect of hippocampal neurons treated with BIA 2-024 (not shown) or BIA 2-093 was similar. Micrographs show neurons stained with $0.5 \%$ Cresyl violet. The black bar in (A-D) represents $50 \mu \mathrm{m}$ and in (E-F) represents $10 \mu \mathrm{m}$. The arrows show condensed and/or fragmented nuclei. 
Carbamazepine, oxcarbazepine, BIA 2-093, BIA 2-024, veratridine and LY 303070 stock solutions were prepared in dimethyl sulfoxide (DMSO).

\subsection{Statistical analysis}

The data are expressed as means \pm S.E.M. Statistical significance was determined by using an analysis of variance (ANOVA), followed by Dunnett's or Bonferroni's post-tests, as indicated in the figure legends.

\section{Results}

\subsection{Effects of antiepileptic drugs on the viability of cul- tured rat hippocampal neurons}

We investigated the effect of $24 \mathrm{~h}$ exposure of cultured hippocampal neurons to BIA 2-093, BIA 2-024, carbamazepine or oxcarbazepine. The presence of $300 \mu \mathrm{M}$ BIA 2-093 for $24 \mathrm{~h}$ decreased the MTT reduction to $84.4 \pm 3.5 \%$ $(P<0.05)$, as compared to control (no drug treatment; Fig. 1A). Lower concentrations of BIA 2-093 (50 and 100 $\mu \mathrm{M})$ did not cause toxicity. In the presence of BIA 2-024 $(300 \mu \mathrm{M})$ the MTT reduction was $86.8 \pm 2.4 \%$ of the control ( $P>0.05$; Fig. 1B). Treatment of hippocampal neurons either with carbamazepine or with oxcarbazepine caused a neurotoxic effect at lower concentrations, as compared to BIA 2-093 or BIA 2-024. In the presence of 100 or $300 \mu \mathrm{M}$ carbamazepine, the MTT reduction decreased to $82.8 \pm 5.3 \%(P<0.05)$ or $75.4 \pm 2.3 \%(P<$ 0.01 ) of the control, respectively (Fig. 1C). The toxic effect caused by oxcarbazepine was even higher. Thus,

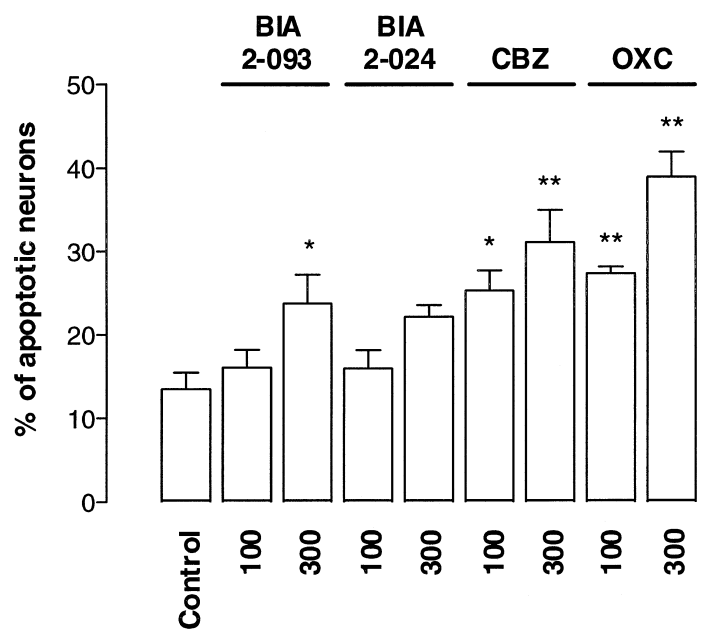

Fig. 3. Quantification of apoptotic hippocampal neurons exposed to antiepileptic drugs for $24 \mathrm{~h}$. The concentration (in $\mu \mathrm{M}$ ) of BIA 2-093, BIA 2-024, carbamazepine (CBZ) or oxcarbazepine (OXC) is indicated below the corresponding bars. The results are presented as percentages of apoptotic neurons, and represent the means \pm S.E.M. of at least three independent experiments. ${ }^{*} P<0.05,{ }^{*}{ }^{*} P<0.01-$ Significantly different from control; Dunnett's post-test.

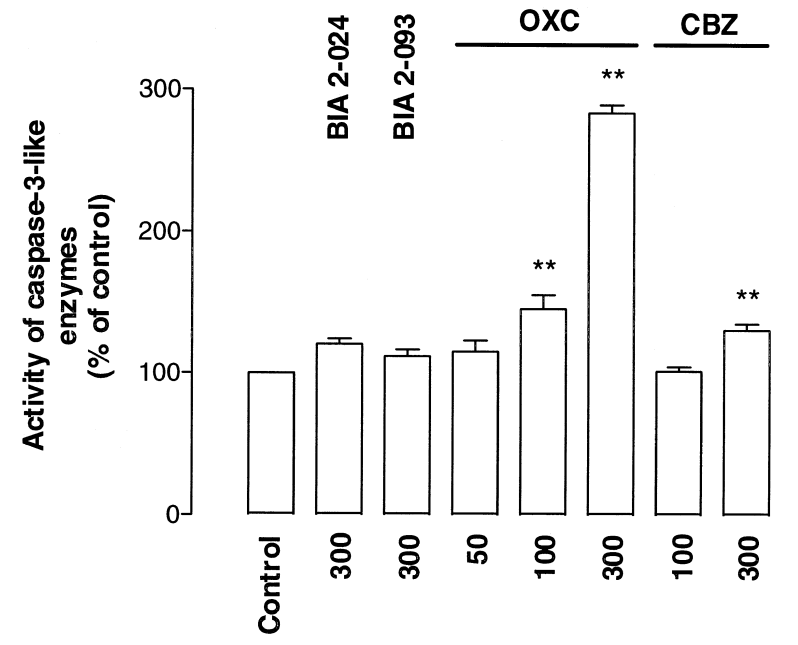

Fig. 4. Activation of caspase-3-like enzymes in cultured hippocampal neurons exposed to antiepileptic drugs. The concentration (in $\mu \mathrm{M}$ ) of BIA 2-024, BIA 2-093, oxcarbazepine (OXC) or carbamazepine (CBZ) is indicated below the corresponding bars. The results are presented as percentages of control (no treatment), using arbitrary fluorescence units at an excitation wavelength of $390 \mathrm{~nm}$, and represent the means \pm S.E.M. of at least three independent experiments. ${ }^{*} P<0.01$ - Significantly different from control; Dunnett's post-test.

treatment of hippocampal neurons with 50,100 or $300 \mu \mathrm{M}$ oxcarbazepine for $24 \mathrm{~h}$ decreased the reduction of MTT to $86.6 \pm 3.9 \%(P<0.05), 65.9 \pm 4.2 \%(P<0.01)$ or 62.5 $\pm 3.2 \%$ of the control, respectively (Fig. 1D). We also exposed hippocampal neurons to the antiepileptic drugs for shorter periods $(1 \mathrm{~h})$ and measured metabolic activity $24 \mathrm{~h}$ later, in order to assess possible delayed neurotoxic effects, but the results indicated that the antiepileptic drugs were not toxic under these conditions (not shown).

Morphological studies showed neuronal deterioration, mainly in hippocampal neurons exposed to high concentrations of carbamazepine or oxcarbazepine (Fig. 2). However, some morphological changes were also observed in neurons treated with high concentrations of either BIA 2-093 or BIA 2-024. In Fig. 2, hippocampal neurons treated with BIA 2-024 are not shown since the morphological changes were similar to those of neurons treated with BIA 2-093. Some neurons showed nuclear condensation, indicative of apoptosis, and other neurons appeared to be disintegrated, suggesting necrotic-like cell death.

We also analysed nuclear condensation/fragmentation in cultured hippocampal neurons exposed to the antiepileptic drugs, by using propidium iodide, which stains nucleic acids in cells pre-fixed with paraformaldehyde. In control cultures, $13 \%$ of total neurons were apoptotic. In the presence of 100 or $300 \mu \mathrm{M}$ BIA 2-093, the proportion of apoptotic neurons was $16 \pm 2 \%$ or $23 \pm 3 \%(P<0.05)$ of total neurons, respectively (Fig. 3). Similar results were obtained with BIA 2-024: the proportion of apoptotic neurons in cultures treated with 100 or $300 \mu \mathrm{M}$ BIA 2-024 was $15 \pm 2 \%$ or $22 \pm 1 \%$ of total neurons, respectively. The number of apoptotic neurons significantly increased in 


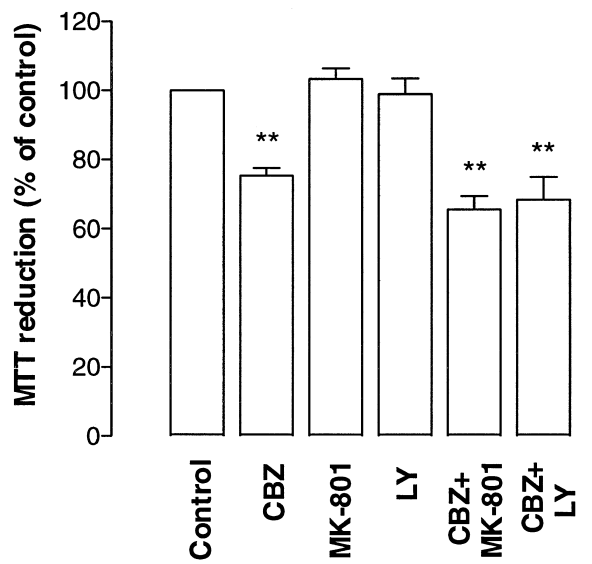

Fig. 5. Lack of protective effect of NMDA or AMPA receptor antagonists on neuronal degeneration caused by carbamazepine (CBZ; $300 \mu \mathrm{M})$. NMDA receptor antagonist-MK-801 $(10 \mu \mathrm{M})$; AMPA receptor antagonist-LY $303070(\mathrm{LY} ; 15 \mu \mathrm{M})$. The results represent the means \pm S.E.M. of at least three independent experiments performed in triplicate, and are presented as percentages of MTT reduction, compared to control conditions (no drug treatment). ${ }^{*} P<0.05,{ }^{*}{ }^{*} P<0.01-$ Significantly different from control; Dunnett's post-test. the presence of either carbamazepine or oxcarbazepine. Thus, when hippocampal neurons were exposed to 100 or $300 \mu \mathrm{M}$ carbamazepine the proportion of apoptotic neurons was $25 \pm 2 \%(P<0.05)$ or $31 \pm 3 \%(P<0.01)$, respectively. In the case of oxcarbazepine (100 or 300 $\mu \mathrm{M})$ the proportion of apoptotic neurons was $27 \pm 1 \%$ $(P<0.01)$ or $39 \pm 3 \%(P<0.01)$ of total neurons, respectively (Fig. 3). These results indicate that the antiepileptic drugs may cause apoptosis, at least at higher concentrations.

\subsection{Effects of antiepileptic drugs on the activity of cas- pase-3-like enzymes}

We measured the activity of caspase- 3 and related proteases in hippocampal neurons treated with antiepileptic drugs by using a fluorogenic substrate for these enzymes. The activity of caspase-3-like enzymes was higher in oxcarbazepine-treated neurons than in neurons treated with the other antiepileptic drugs (Fig. 4). The presence of BIA
A

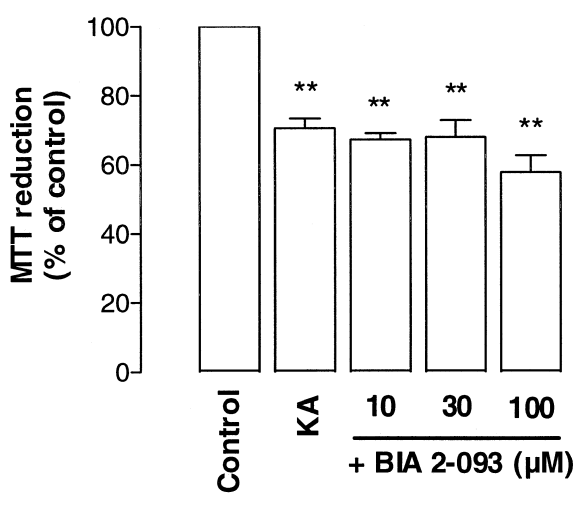

C

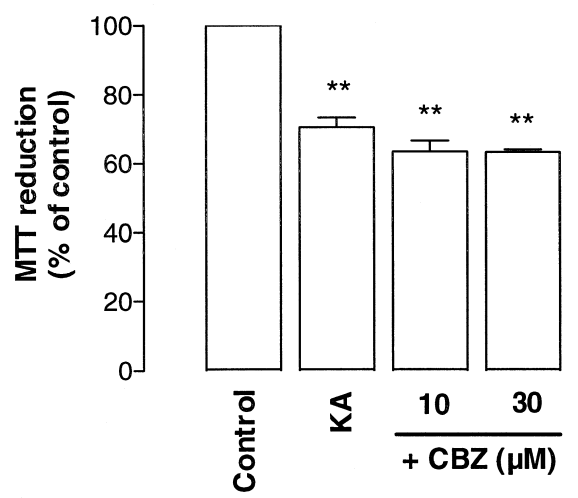

B
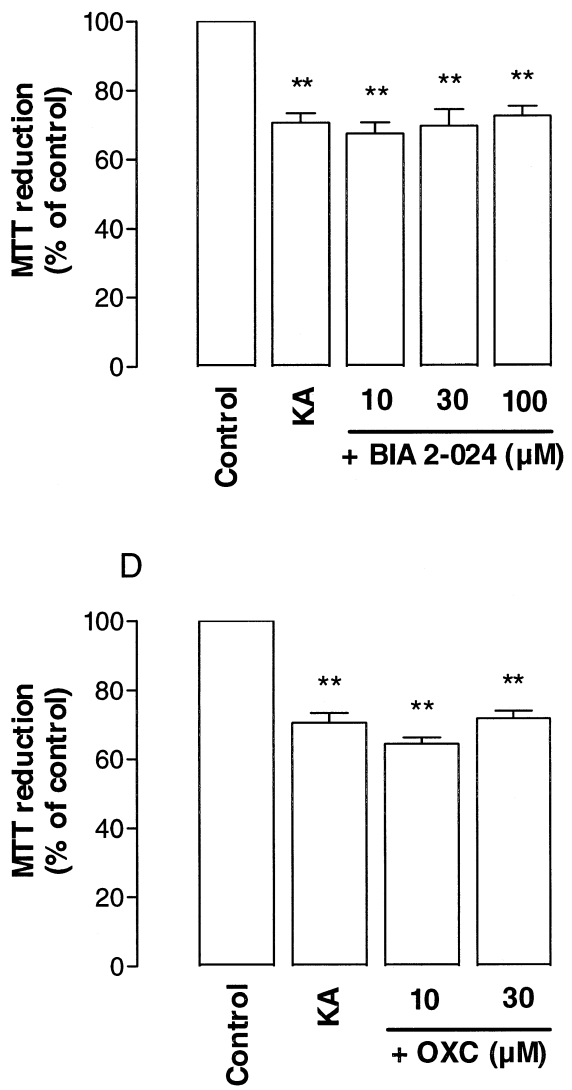

Fig. 6. Lack of protective effect of BIA 2-093, BIA 2-024, carbamazepine (CBZ) or oxcarbazepine (OXC) on neuronal degeneration caused by exposure of hippocampal neurons to kainate $(\mathrm{KA} ; 100 \mu \mathrm{M})$ for $24 \mathrm{~h}$. The concentrations of the antiepileptic drugs are indicated below the bars. The results represent the means \pm S.E.M. of at least three independent experiments performed in triplicate, and are presented as percentages of MTT reduction under control conditions (no drug treatment). ${ }^{*} P<0.01$ - Significantly different from control; Dunnett's post-test. 
2-024 (300 $\mu \mathrm{M})$ or BIA 2-093 (300 $\mu \mathrm{M})$ increased the activity of caspase-3-like enzymes to only $120.7 \pm 3.8 \%$ $(P>0.05)$ or $112.1 \pm 4.8 \%(P>0.05)$ of the control, respectively. However, oxcarbazepine and carbamazepine caused a higher and significant increase, especially at high concentrations. Thus, oxcarbazepine (50, 100 and 300 $\mu \mathrm{M})$ increased the activity of caspase-3-like proteases to $115.4 \pm 7.9 \%(P>0.05), 145.8 \pm 9.8 \%$ and $283.9 \pm 5.6 \%$ of the control, respectively. In the case of carbamazepine (300 $\mu \mathrm{M}$ ), the activity of caspase-3-like enzymes increased to $130.9 \pm 4.7 \%$ of the control, and no effect was observed at $100 \mu \mathrm{M}$.

\subsection{Effects of $N$-methyl-D-aspartate (NMDA) or $\alpha$-amino-} 3-hydroxy-5-methyl-isoxazole-4-propionate (AMPA) receptor antagonists on the toxicity caused by carbamazepine

We also checked whether NMDA or AMPA receptors are involved in the neurotoxic effect caused by carbamazepine. For this purpose, we treated hippocampal neurons with a high concentration of carbamazepine (300 $\mu \mathrm{M})$ in the presence of either MK-801 (10 $\mu \mathrm{M})$, a NMDA receptor antagonist, or LY $303070(15 \mu \mathrm{M})$, an AMPA receptor antagonist, and observed that these glutamate receptor antagonists did not protect neurons from the toxicity caused by carbamazepine (Fig. 5). Similar results were obtained in neurons treated with oxcarbazepine (not shown), suggesting that NMDA and AMPA receptors are not involved in the toxicity caused by carbamazepine or oxcarbazepine.

3.4. Kainate-induced neurotoxicity in cultured hippocampal neurons: lack of protection by BIA 2-093, BIA 2-024, carbamazepine or oxcarbazepine

Non-NMDA receptor activation contributes to the epileptic phenomena and is also implicated in excitotoxicity. Thus, we investigated whether BIA 2-093, BIA 2-024, carbamazepine and oxcarbazepine act as neuroprotectors against the neurotoxic effect caused by kainate. Treatment of cultured hippocampal neurons with $100 \mu \mathrm{M}$ kainate for $24 \mathrm{~h}$ decreased MTT reduction to $70.5 \pm 2.9 \%$ of the control (Fig. 6). As previously shown, this neurotoxic effect was mainly due to the activation of AMPA receptors
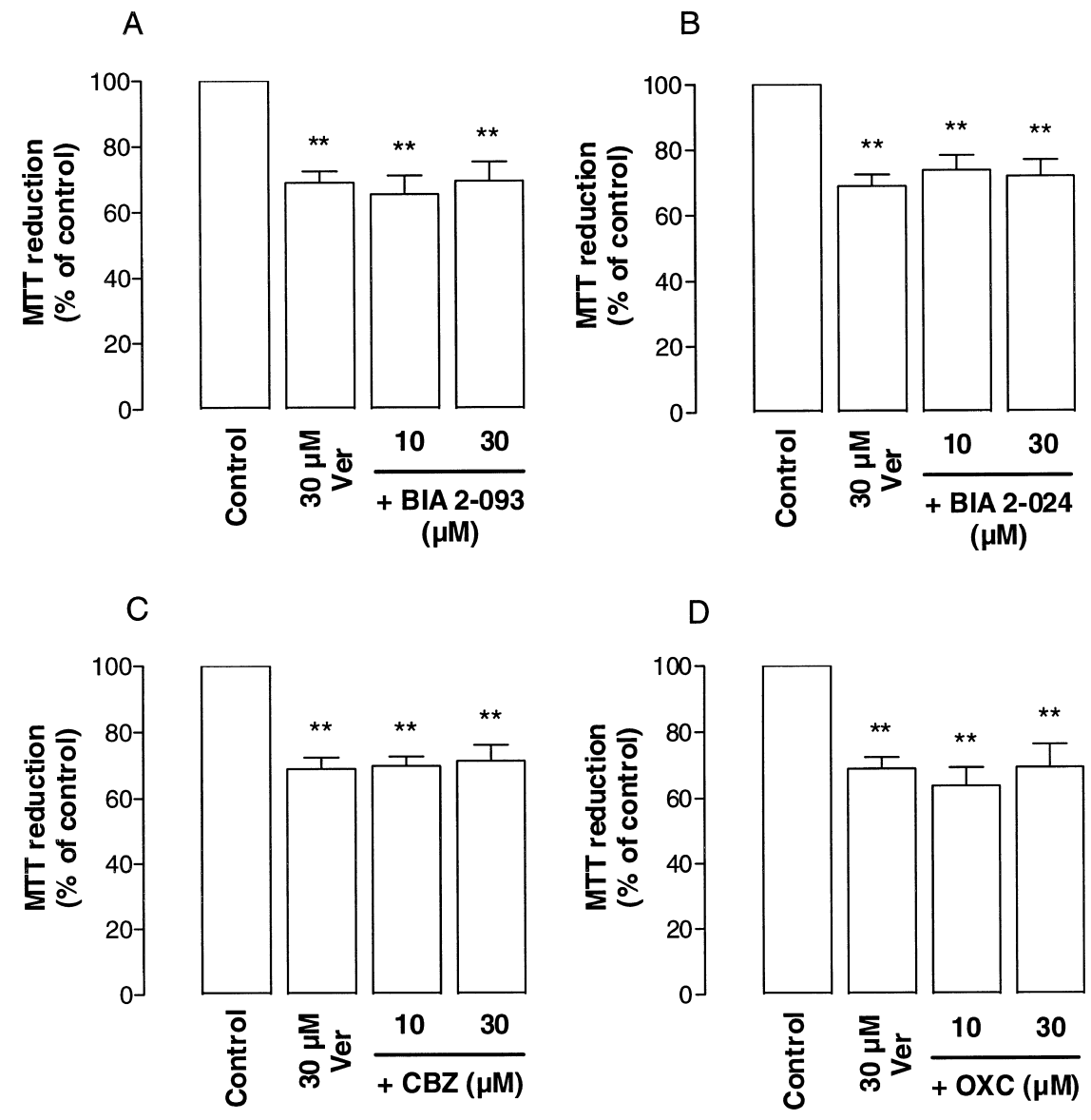

Fig. 7. Lack of protective effect of BIA 2-093, BIA 2-024, carbamazepine (CBZ) or oxcarbazepine (OXC) on neuronal degeneration caused by veratridine (Ver; $30 \mu \mathrm{M})$. The results represent the means \pm S.E.M. of at least three independent experiments performed in triplicate, and are presented as percentages of MTT reduction under control conditions (no drug). ${ }^{* *} P<0.01-$ Significantly different from control; Dunnett's post-test. 
(Ambrósio et al., 2000). The presence of BIA 2-093, BIA 2-024, carbamazepine or oxcarbazepine, at concentrations below those that did not cause any toxic effect, did not protect neurons from kainate-induced toxicity (Fig. 6).

\subsection{Veratridine-induced neurotoxicity: lack of protection} by BIA 2-093, BIA 2-024, carbamazepine or oxcarbazepine

The antiepileptic drugs BIA 2-093 (Benes et al., 1999a), BIA 2-024 (unpublished data), carbamazepine and oxcarbazepine (McLean et al., 1994; Kuo et al., 1997; Benes et al., 1999a) are known to block voltage-sensitive sodium channels. We investigated whether these antiepileptic drugs could protect hippocampal neurons against veratridine-induced toxicity. Veratridine caused a concentration-dependent neurotoxic effect. We observed that in hippocampal neurons treated for $24 \mathrm{~h}$ with 10,30 or $50 \mu \mathrm{M}$ veratridine the MTT reduction decreased to $85.6 \pm 1.8 \%(P>0.05)$, $68.9 \pm 3.5 \%(P<0.01)$ or $49.7 \pm 2.3 \%$ of the control, respectively. In order to test the efficacy of the antiepileptic drugs in protecting hippocampal neurons, we chose the lowest concentration of veratridine that caused a significant neurotoxic effect: $30 \mu \mathrm{M}$. The results obtained show that the antiepileptic drugs, at concentrations below those that caused toxicity, did not protect hippocampal neurons from veratridine-induced toxicity (Fig. 7).

3.6. Chemical ischaemia-induced toxicity: lack of protection by BIA 2-093, BIA 2-024, carbamazepine or oxcarbazepine

Some reports indicate that antiepileptic drugs may be neuroprotective under ischaemic conditions (Rataud et al., 1994; Taylor, 1996). We further investigated a possible neuroprotective role of BIA 2-093, BIA 2-024, carbamazepine or oxcarbazepine, by testing their effects in hippocampal neurons exposed to ischaemia-like conditions. Exposure of hippocampal neurons to both $5 \mathrm{mM}$ 2-deoxyglucose and $2.5 \mathrm{mM}$ sodium cyanide, for $10 \mathrm{~min}$, decreased the MTT reduction to $49.0 \pm 3.7 \%$ of the control (Fig. 8A). After washing and a further 1-h incubation in Neurobasal medium, neurons partially recovered their metabolic activity, since the MTT reduction increased to $69.3 \pm 4.1 \%$ of the control $\left({ }^{++} P<0.01\right.$, as compared to that after a 10 -min exposure to deoxyglucose plus cyanide). However, $2 \mathrm{~h}$ after exposure to ischaemia-like conditions, an impairment of metabolic activity was observed, since the MTT reduction was similar $(54.2 \pm 3.3 \%$ of the control) to that observed when neurons were treated with deoxyglucose and cyanide for $10 \mathrm{~min}$, without a recovery period. Moreover, when neurons were treated with either deoxyglucose or cyanide, and left to recover for $2 \mathrm{~h}$, the MTT reduction was $83.6 \pm 3.6 \%(P<0.05)$ or $86.8 \pm$ $4.9 \%(P>0.05)$ of the control, respectively (Fig. 8A).

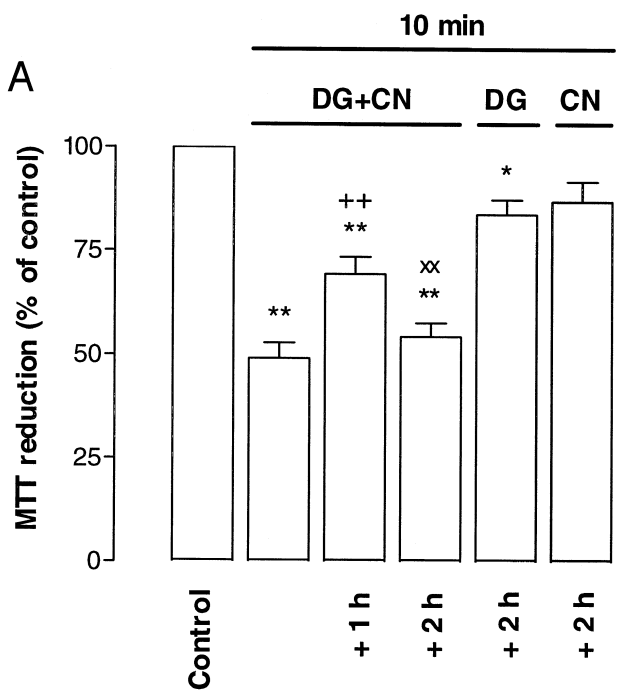

B

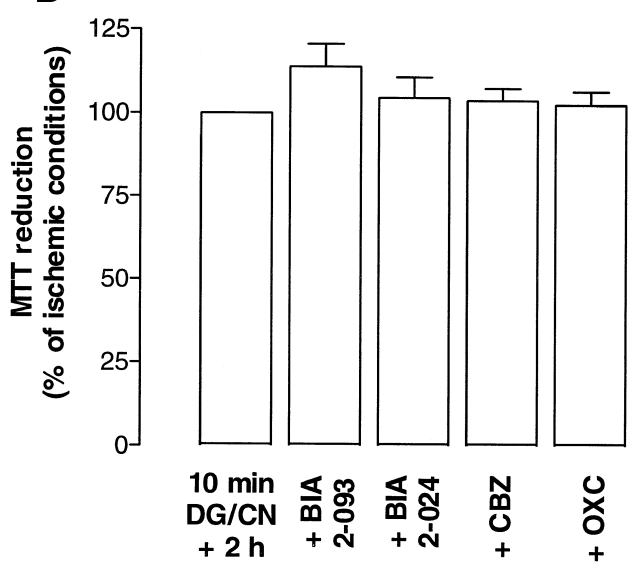

Fig. 8. (A) Evaluation of the metabolic activity of hippocampal neurons exposed to ischaemia-like conditions. Hippocampal neurons were treated with $5 \mathrm{mM}$ 2-deoxyglucose (DG) and/or $2.5 \mathrm{mM}$ sodium cyanide (CN) for $10 \mathrm{~min}$. As indicated below bars, in some cases neurons were left to recover for $1 \mathrm{~h}$ or $2 \mathrm{~h}$, respectively. The results represent the means \pm S.E.M. of at least three independent experiments performed in triplicate, and are presented as percentage of MTT reduction, compared to that under control conditions (no drug). ${ }^{*} P<0.05,{ }^{*}{ }^{*} P<0.01-$ Significantly different from control; Dunnett's post-test. ${ }^{++} P<0.01-$ Significantly different from chemical-ischaemia conditions; ${ }^{\mathrm{xx}} P<0.01-$ Significantly different from chemical-ischaemia conditions plus $1 \mathrm{~h}$ recovery in Neurobasal medium; Bonferroni's post-test. (B) Lack of protective effect of BIA 2-093, BIA 2-024, carbamazepine (CBZ) or oxcarbazepine (OXC) against ischaemia-like conditions in cultured hippocampal neurons. In this particular case, ischaemic conditions were ischaemia-like conditions plus $2 \mathrm{~h}$ recovery in Neurobasal medium, in the absence of antiepileptic drugs. In non-control conditions the antiepileptic drugs (300 $\mu \mathrm{M})$ were present during both ischaemic and recovery periods. The results represent the means \pm S.E.M. of at least six independent experiments performed in triplicate, and are presented as percentage of MTT reduction, compared to that under ischaemic conditions.

We then investigated the effect of BIA 2-093, BIA 2-024, carbamazepine or oxcarbazepine (at $300 \mu \mathrm{M}$ ) on the metabolic activity of hippocampal neurons exposed to ischaemia-like conditions. The antiepileptic drugs were present when hippocampal neurons were exposed for 10 
min to both deoxyglucose and cyanide, as well as during the 2 -h recovery period. We chose a concentration of the antiepileptic drugs $(300 \mu \mathrm{M})$ which was not toxic to hippocampal neurons after a 2-h exposure. The results show that the presence of the antiepileptic drugs did not protect hippocampal neurons from the toxic effect caused by ischaemia-like conditions (Fig. 8B).

\section{Discussion}

\subsection{Neurotoxic effect caused by antiepileptic drugs in cultured hippocampal neurons}

It is widely accepted that antiepileptic drugs may cause CNS side effects. For example, antiepileptic drug therapy can affect cognitive function in patients with epilepsy (Delcker et al., 1997; Meador, 1998). Conversely, it has been shown that some antiepileptic drugs have neuroprotective effects (Rataud et al., 1994; Minato et al., 1997). In the present work, we used an in vitro preparation to investigate the neurotoxic/neuroprotective profile of two new anticonvulsants, BIA 2-093 and BIA 2-024, in comparison to the established compounds carbamazepine and oxcarbazepine.

We found that BIA 2-093 and BIA 2-024 were less toxic to hippocampal neurons than are the related compounds, carbamazepine and oxcarbazepine, based on the fact that carbamazepine and oxcarbazepine are more toxic at lower concentrations. Indeed, at the highest concentration used (300 $\mu \mathrm{M})$, BIA 2-024 did not cause a significant toxic effect, as assessed either by MTT assay or by propidium iodide staining. However, carbamazepine and oxcarbazepine were toxic, although at concentrations above those found in the CSF or plasma. For example, in humans, therapeutic serum levels of carbamazepine are 17-51 $\mu \mathrm{M}$, and CSF levels may range from $17 \%$ to $31 \%$ of the plasma concentration (Rogawski and Porter, 1990). Moreover, in rats receiving four times the $\mathrm{ED}_{50}$ dose of carbamazepine against maximal electroshock seizure, the peak brain extracellular fluid concentration is approximately 11 $\mu \mathrm{M}$ (Dailey et al., 1997). However, the concentrations used in the present work were not different from the concentrations reported previously (Stefani et al., 1995; Dailey et al., 1997; Nonaka et al., 1998; Benes et al., 1999a; Lingamaneni and Hemmings, 1999). The toxic effect observed at high concentrations may be relevant to some of the adverse syndromes associated with overdosage.

It is important to mention that the antiepileptic drugs studied are not metabolised by neuronal tissue in vitro (unpublished observations), but are rapidly metabolised in vivo, giving origin to various metabolites, some of which are also responsible for the effect of the drugs (Rogawski and Porter, 1990; unpublished observations). Thus, it is difficult to extrapolate these results to an in vivo situation.
However, these results may be useful to better understand the mechanism of action of antiepileptic drugs, or even to compare their neurotoxic/neuroprotective profile, since it is important to find new antiepileptic drugs with fewer CNS adverse effects.

Exposure of hippocampal neurons to high concentrations of the antiepileptic drugs caused apoptosis, mainly in the case of oxcarbazepine and carbamazepine, and the apoptotic pathway may involve the activation of caspase3-like enzymes. It was previously shown that exposure of cultured cerebellar granule cells to carbamazepine for 3 days induced apoptosis (Gao and Chuang, 1992; Gao et al., 1995; Nonaka et al., 1998). Some antiepileptic drugs, including carbamazepine, were found to reduce glutamine synthetase activity in mouse brain (Fraser et al., 1999), and acute CNS side effects in healthy volunteers were also reported (Noachtar et al., 1998). Oxcarbazepine is a new antiepileptic drug that is almost clinically indistinguishable from carbamazepine, but which has some improved properties, as for example in terms of liver enzyme-inducing effects (Elger and Bauer, 1998). To our knowledge, there are no reports in the literature concerning toxic effects of this carbamazepine derivative in the CNS. Surprisingly, we observed that oxcarbazepine was more toxic than carbamazepine. The toxic effect of oxcarbazepine was more pronounced and was observed at lower concentrations.

It is well established that excessive activation of ionotropic glutamate receptors causes neurodegeneration. However, NMDA or AMPA receptors were not involved in the neurotoxic effect caused by high concentrations of carbamazepine, since the toxic effect was not prevented by NMDA or AMPA receptor antagonists.

BIA 2-093 was found to be as potent as carbamazepine, and more potent than oxcarbazepine, in preventing maximal electroshock-induced seizures, when the compounds were given by gastric tube. Both BIA 2-093, carbamazepine and oxcarbazepine caused a dose-dependent motor impairment in rats. When the compounds were given by intraperitoneal route, the protective index for BIA 2-093 was greater than the protective index for carbamazepine and oxcarbazepine (Benes et al., 1999a). Thus, these results, together with our findings, suggest that BIA 2-093 or BIA 2-024 may have some advantages as compared to carbamazepine or oxcarbazepine.

4.2. Toxicity induced by kainate, veratridine or ischaemia-like conditions: lack of protection by BIA 2-093, BIA 2-024, carbamazepine or oxcarbazepine

Glutamate is involved in the initiation of seizures and their propagation, and some evidence indicates potential roles for both NMDA and non-NMDA receptors (Löscher, 1998b). Moreover, kainic acid seizure models are widely used to evaluate the efficacy of antiepileptic agents. Taking this into account, we investigated whether BIA 2-093, BIA 2-024, carbamazepine or oxcarbazepine, at concentra- 
tions that did not cause toxicity, could have a neuroprotective effect on kainate-induced toxicity in hippocampal neurons, which is essentially mediated by the activation of AMPA receptors (Ambrósio et al., 2000). The results show that the antiepileptic drugs did not protect hippocampal neurons from the toxicity caused by kainate.

Contrary to our results, it was previously reported that carbamazepine afforded significant protection against glutamate neurotoxicity in hippocampal cell cultures (Mattson and Kater, 1989; Mark et al., 1995), and reduced NMDAmediated brain injury (McDonald and Johnston, 1990). It was suggested that the neuroprotective mechanism involved stabilisation of $\left[\mathrm{Ca}^{2+}\right]_{i}$. These findings point to beneficial effects of antiepileptic drugs against ionotropic glutamate receptor-mediated injury; however, in our system, the antiepileptic drugs failed to protect hippocampal neurons against toxicity mediated by glutamate receptors. We found previously that carbamazepine inhibited kainate-induced $\left[\mathrm{Ca}^{2+}\right]_{\mathrm{i}}$ elevation (Ambrósio et al., 1999), but probably this effect was not enough to rescue neurons from kainate-induced toxicity. We also found that ionotropic glutamate receptors, both NMDA and nonNMDA, were not affected by carbamazepine (Ambrósio et al., 1999). Moreover, Grant et al. (1992) showed that the inhibition caused by carbamazepine of NMDA-induced responses appeared to be independent of the NMDA recognition site. These findings suggest that blockade of ionotropic glutamate receptors is not the mechanism by which carbamazepine exerted protective effects in other systems.

Since BIA 2-093, BIA 2-024, carbamazepine or oxcarbazepine inhibit voltage-sensitive sodium channels (McLean et al., 1994; Kuo et al., 1997; Benes et al., 1999a; unpublished observations), we investigated whether these antiepileptic drugs could be neuroprotective against veratridine-induced toxicity. The results showed that the antiepileptic drugs did not protect hippocampal neurons from veratridine-induced toxicity. Probably, the concentrations of the antiepileptic drugs were not high enough to inhibit the effect of veratridine. However, it was previously reported that some antiepileptic drugs, including carbamazepine, protected rat cortical cultures against 100 $\mu \mathrm{M}$ veratridine-induced cell death (Lakics et al., 1995). In addition, these authors suggested that mechanisms other than sodium channel blockade may be involved in the neuroprotection.

There is now substantial evidence from animal models that sodium channel blockers prevent neuronal damage caused by global and focal brain ischaemia (Taylor, 1996). We used an ischaemic-like insult (chemical ischaemia) in order to evaluate whether BIA 2-093, BIA 2-024, carbamazepine or oxcarbazepine has a neuroprotective effect in hippocampal neurons. The effect of 2-deoxyglucose and cyanide was synergistic, because exposure of hippocampal neurons to either deoxyglucose or cyanide alone caused only a small toxic effect. The presence of the antiepileptic drugs, during and after the ischaemia-like insult, did not protect hippocampal neurons. Conversely, in vivo studies have demonstrated that some antiepileptic drugs, including carbamazepine, reduce cerebral damage after focal ischaemia in rodents (Rataud et al., 1994; Minato et al., 1997), pointing to a therapeutic potential for voltage-dependent sodium channel blockers. Such compounds may act at sodium channels to prevent depolarisation, inhibiting the release of neurotransmitters such as glutamate and thus protecting the brain. Minato et al. (1997) also showed that carbamazepine ameliorated the effects of brain infarction and improved neurological deficits, but only above the anticonvulsant dose. Contrary to this observation, it was also shown that carbamazepine protected the rat optic nerve against anoxic injury at concentrations well below those used clinically to treat epilepsy (Fern et al., 1993).

Taken together, our results showed that the antiepileptic drugs tested may be toxic to cultured hippocampal neurons, at least at high concentrations, and that the toxic effect is also observed as apoptosis. However, the new putative antiepileptic drugs, BIA 2-093 and BIA 2-024, were less toxic than carbamazepine or oxcarbazepine, with oxcarbazepine being the most toxic. The activation of NMDA or AMPA receptors did not contribute to the toxic effect caused by carbamazepine. In addition, these antiepileptic drugs failed to protect hippocampal neurons against different toxic insults: kainate or veratridine exposure, and ischaemia-like conditions. However, previous findings for other systems, indicate that antiepileptic drugs, under some conditions, can be neuroprotective.

\section{Acknowledgements}

We would like to thank Ms. Elisabete Carvalho for assisting us with the hippocampal cell cultures. This work was supported by Fundação para a Ciência e a Tecnologia, Programa PRAXIS XXI, Portugal (Grant PRAXIS XXI $5604 / 95)$, and by BIAL, Portugal.

\section{References}

Ambrósio, A.F., Silva, A.P., Malva, J.O., Mesquita, J.F., Carvalho, A.P., Carvalho, C.M., 2000. Role of desensitization of AMPA receptors on the neuronal viability and on the $\left[\mathrm{Ca}^{2+}\right]_{\mathrm{i}}$ changes in cultured hippocampal neurons. Eur. J. Neurosci. 12, 2021-2031.

Ambrósio, A.F., Silva, A.P., Malva, J.O., Soares-da-Silva, P., Carvalho, A.P., Carvalho, C.M., 1999. Carbamazepine inhibits L-type $\mathrm{Ca}^{2+}$ channels in cultured hippocampal neurons stimulated with glutamate receptor agonists. Neuropharmacology 38, 1349-1359.

Benes, J., Parada, A., Figueiredo, A.A., Alves, P.C., Freitas, A.P., Learmonth, D.A., Cunha, R.A., Garrett, J., Soares-da-Silva, P., 1999a. Anticonvulsant and sodium channel-blocking properties of novel 10,11-dihydro-5 $H$-dibenz $[b, f]$ azepine-5-carboxamide derivatives. J. Med. Chem. 42, 2582-2587.

Benes, J., Soares-Da-Silva, P., Learmonth, D.A., 1999b. Derivatives of 10,11-dihydro-10-oxo-5H-dibenz[ $b, f]$ azepine-5-carboxamide. US Patent. Patent Number 5,866,566. 
Brewer, G.J., Torricelli, J.R., Evege, E.K., Price, P.J., 1993. Optimized survival of hippocampal neurons in B27-supplemented Neurobasal ${ }^{\mathrm{TM}}$, a new serum-free medium combination. J. Neurosci. Res. 35, 567-576.

Dailey, J.W., Reith, M.E.A., Yan, Q.S., Li, M.Y., Jobe, P.C., 1997. Carbamazepine increases extracellular serotonin concentration: lack of antagonism by tetrodotoxin or zero $\mathrm{Ca}^{2+}$. Eur. J. Pharmacol. 328, $153-162$.

Delcker, A., Wilhelm, H., Timmann, D., Diener, D., 1997. Side effects from increased doses of carbamazepine on neuropsychological and posturographic parameters of humans. Eur. Neuropsychopharmacol. 7, 213-218.

Elger, C.E., Bauer, J., 1998. New antiepileptic drugs in epileptology. Neuropsychobiology 38, 145-148.

Fern, R., Ransom, B.R., Stys, P.K., Waxman, S.G., 1993. Pharmacological protection of CNS white matter during anoxia: actions of phenytoin, carbamazepine and diazepam. J. Pharmacol. Exp. Ther. 266, $1549-1555$

Fraser, C.M., Sills, G.J., Forrest, G., Thompson, G.G., Brodie, M.J., 1999. Effects of anti-epileptic drugs on glutamine synthetase activity in mouse brain. Br. J. Pharmacol. 126, 1634-1638.

Gao, X.-M., Chuang, D.-M., 1992. Carbamazepine-induced neurotoxicity and its prevention by NMDA in cultured cerebellar granule cells. Neurosci. Lett. 135, 159-162.

Gao, X.-M., Margolis, R.L., Leeds, P., Hough, C., Post, R.M., Chuang, D.-M., 1995. Carbamazepine induction of apoptosis in cultured cerebellar neurons: effects of $N$-methyl-D-aspartate, aurintricarboxylic acid and cycloheximide. Brain Res. 703, 63-71.

Grant, K.A., Snell, L.D., Rogawski, M.A., Thurkauf, A., Tabakoff, B., 1992. Comparison of the effects of the uncompetitive $N$-methyl-Daspartate antagonist ( \pm )-5-aminocarbonyl-10,11-dihydro- $5 \mathrm{H}$-dibenzo[ $a, d]$ cyclohepten-5,10-imine (ADCI) with its structural analogue dizocilpine (MK-801) and carbamazepine on ethanol withdrawal seizures. J. Pharmacol. Exp. Ther. 260, 1017-1022.

Kaneko, S., Battino, D., Andermann, E., Wada, K., Kan, R., Takeda, A., Nakane, Y. et al., 1999. Congenital malformations due to antiepileptic drugs. Epilepsy Res. 33, 145-158.

Kuo, C.C., Chen, R.S., Lu, L., Chen, R.C., 1997. Carbamazepine inhibition of neuronal $\mathrm{Na}^{+}$currents - quantitative distinction from phenytoin and possible therapeutic implications. Mol. Pharmacol. 51, 10771083.

Lakics, V., Molnar, P., Erdo, S.L., 1995. Protection against veratridine toxicity in rat cortical cultures: relationship to sodium channel blockade. Neuroreport 7, 89-92.

Lingamaneni, R., Hemmings, H.C. Jr, 1999. Effects of anticonvulsants on veratridine- and $\mathrm{KCl}$-evoked glutamate release from rat cortical synaptosomes. Neurosci. Lett. 276, 127-130.

Loiseau, P., Duché, P., 1995. Carbamazepine. Clinical use. In: Levy, R.H., Mattson, R.H., Meldrum, M.S. (Eds.), Antiepileptic Drugs. Raven Press, New York, NY, pp. 555-566.

Löscher, W., 1998a. New visions in the pharmacology of anticonvulsion. Eur. J. Pharmacol. 342, 1-13.

Löscher, W., 1998b. Pharmacology of glutamate receptor antagonists in the kindling model of epilepsy. Prog. Neurobiol. 54, 721-741.
Mark, R.J., Ashford, J.W., Goodman, Y., Mattson, M.P., 1995. Anticonvulsants attenuate amyloid $\beta$-peptide neurotoxicity, $\mathrm{Ca}^{2+}$ deregulation, and cytoskeletal pathology. Neurobiol. Aging 16, 187-198.

Mattson, M.P., Kater, S.B., 1989. Excitatory and inhibitory neurotransmitters in the generation and degeneration of hippocampal neuroarchitecture. Brain Res. 478, 337-348.

McDonald, J.W., Johnston, M.V., 1990. Pharmacology of $N$-methyl-Daspartate-induced brain injury in an in vivo perinatal rat model. Synapse 6, 179-188.

McLean, M.J., Schmutz, M., Wamil, A.W., Olpe, H.R., Portet, C., Fedmann, K.F., 1994. Oxcarbazepinearbazepine: mechanisms of action. Epilepsia 35 (Suppl. 35), 55-59.

Meador, K.J., 1998. Cognitive effects of the new antiepileptic drugs. Neurologist 4, S35-S39.

Minato, H., Kikuta, C., Fujitani, B., Masuda, Y., 1997. Protective effect of zonisamide, an antiepileptic drug, against transient focal cerebral ischemia with middle cerebral artery occlusion-reperfusion in rats. Epilepsia 38, 975-980.

Noachtar, S., von Maydell, B., Fuhry, L., Büttner, U., 1998. Gabapentin and carbamazepine affect eye movements and posture control differently: a placebo-controlled investigation of acute CNS side effects in healthy volunteers. Epilepsy Res. 31, 47-57.

Nonaka, S., Katsube, N., Chuang, D.-M., 1998. Lithium protects rat cerebellar granule cells against apoptosis induced by anticonvulsants, phenytoin and carbamazepine. J. Pharmacol. Exp. Ther. 286, 539-547.

Pitkänen, A., Tuunanen, J., Halonen, T., 1996. Vigabatrin and carbamazepine have different efficacies in the prevention of status epilepticus induced neuronal damage in the hippocampus and amygdala. Epilepsy Res. 24, 29-45.

Rataud, J., Debarnot, F., Véronique, M., Pratt, J., Stutzmann, J.-M., 1994. Comparative study of voltage-sensitive sodium channel blockers in focal ischaemia and electric convulsions in rodents. Neurosci. Lett. 172, 19-23.

Rogawski, M.A., Porter, R.J., 1990. Antiepileptic drugs: pharmacological mechanisms and clinical efficacy with consideration of promising developmental stage compounds. Pharmacol. Rev. 42, 223-286.

Scheuer, M.L., Pedley, T.A., 1990. The evaluation and treatment of seizures. N. Eng. J. Med. 323, 1468-1474.

Stefani, A., Pisani, A., De Murtas, M., Mercuri, N.B., Mercuri, N.B., Marciani, M.G., Calabresi, P., 1995. Action of GP 47779, the active metabolite of oxcarbazepinearbazepine, on the corticostriatal system: II. Modulation of high-voltage-activated calcium currents. Epilepsia 336, 997-1002.

Tateishi, T., Asoh, M., Nakura, H., Watanabe, M., Tanaka, M., Kumai, T., Kobayashi, S., 1999. Carbamazepine induces multiple cytochrome P450 subfamilies in rats. Chem. Biol. Int. 117, 257-268.

Taylor, C.P., 1996. Voltage-gated $\mathrm{Na}^{+}$channels as targets for anticonvulsant, analgesic and neuroprotective drugs. Curr. Pharm. Des. 2, 375-388.

Yasui, N., Otani, K., Kaneko, S., Shimoyama, R., Ohkubo, T., Sugawara, K., 1997. Carbamazepine toxicity induced by clarithromycin coadministration in psychiatric patients. Int. Clin. Psychopharmacol. 12, 225229. 